\title{
Mismatch Strain Programmed Shape Transformation of Curved Bilayer-Flexible Support Assembly
}

\author{
Arif M. Abdullah ${ }^{a}$, Kewang Nan ${ }^{a}$, John A. Rogers ${ }^{b, c}$, K. Jimmy Hsia ${ }^{a, b, d^{*}}$ \\ ${ }^{a}$ Department of Mechanical Science and Engineering, University of Illinois at Urbana-Champaign, \\ Urbana, IL 61801 \\ ${ }^{\mathrm{b}}$ Frederick Seitz Materials Research Laboratory, University of Illinois at Urbana-Champaign, \\ Urbana, IL 61801 \\ ${ }^{c}$ Department of Materials Science and Engineering, University of Illinois at Urbana-Champaign, \\ Urbana, IL 61801 \\ ${ }^{\mathrm{d}}$ Departments of Mechanical Engineering and Biomedical Engineering, Carnegie Mellon University, \\ Pittsburgh, PA 15213 \\ "To whom correspondence may be addressed. Email: kjhsia@cmu.edu
}

\begin{abstract}
Shape transformation in three dimensional (3D) structures is of interest in the design of engineered systems capable of accomplishing particular tasks that are unachievable by two dimensional (2D) architectures or static 3D ones. One approach involves the incorporation of stimuli responsive materials into the structural assembly to induce such transformations. In this work, we investigate the transformation of a curved bilayer ribbon supported by a flexible assembly that belongs to a family of complex three dimensional architectures. Through finite element analysis, we identified key design parameters and their effects on the deformation behavior of the assembly when it is subjected to an external stimuli in the form of a mismatch strain. Our results show that the behavior of the curved bilayer in response to the stimuli could be tuned by controlling the structural properties of the assembly. Our calculations also reveal a diverse set of deformation mechanisms including gradual flipping, snapping and creasing of the curved bilayer under specific circumstances. The design principles established in this work could be used to engineer 3D sensors, actuators for traditional and soft robotics, electronic device components, metamaterials, energy storage and harvesting devices with on-demand functional capabilities enabled by $3 \mathrm{D}$ transformations.
\end{abstract}

Keywords: Programmable matter, Mechanical instability, 3D architecture, Shape transformation, Finite element analysis.

(C) 2016. This manuscript version is made available under the Elsevier user license http://www.elsevier.com/open-access/userlicense/1.0/ 


\section{Introduction}

Plants, despite lacking muscle cells, are capable of shape transformation in response to a diverse set of environmental stimuli for their growth, nutrition, reproduction, acclimatization and defense across multiple time and length scales. Specific examples include helical coiling of cucumber tendrils (Cucumis sativus) attached to a support [1], fast closing of swollen bladderwort (Utricularia inflate) traps [2], propelling motion of wild wheat (Triticum turgidum) awns into the ground [3], folding of Easter lily (Lilium longiflorum) pollen wall [4] and periodic closing-opening of shy plant (Mimosa pudica) leaves [5]. One particular design principle through which plants achieve stimuli responsive organ actuation is the utilization of bilayer structures consisting of composites [6]. These composites are reinforced along different directions and hence demonstrate different swelling-shrinking behavior during the inflow and outflow of water. These differences introduce mismatch strains that cause the bilayer to deform in the presence/ absence of water. This basic idea of mismatch strain driven shape formation has been exploited to demonstrate stimuli responsive, programmable complex shape transformation behavior with geometries as diverse as cubes, pyramids, flowers, stars, helices, tubes and origami patterns [7]-[14]. Materials responsive to a variety of external stimuli play central roles in translating biomimetic design principles into functional shape transforming structures. Research has been done on metals, semiconductors and polymers such as $\mathrm{Cr}, \mathrm{SiO}_{\mathrm{x}}, \mathrm{SiGe}, \mathrm{SiN}_{\mathrm{x}}, \mathrm{PDMS}$, Epoxy, Polystyrene, PNIPAM gel and liquid crystalline polymer networks [9], [12], [14]-[16] for realizing mismatch strain driven shape formation.

One specific structure of interest for stimuli responsive shape transformation would be curved beams attached to flexible supports. This class of structures has recently been fabricated [17], [18] from lithographically patterned planar precursors. Here, buckling, which is an elastic instability, enables the formation of functional complex three dimensional architectures. Since elasticity equations are independent of geometry and materials, it is possible, as demonstrated by the authors, to tune the spatial configurations and constituent material properties of the fabricated structures. Due to the unique, scalable nature of the fabrication procedure, shapes that are otherwise difficult to achieve can be realized and applied to different material systems. As such, these concepts are well poised for applications in 3D devices across diverse technological disciplines. In this work, we computationally investigated the stimuli responsive shape transformation behavior of these structures. We adopted a particular design referred to previously as a triple-floor building [17] as the starting point of our work. As shown in Figure 1(a), this structure consists of a curved beam at the highest level and its ends are attached to multilevel wavy ribbons that essentially work as elastic flexible supports. We envisioned a scenario where the curved beam is a bilayer capable of inversion in response to external stimuli (a detailed step by step fabrication scheme is discussed in the Supplementary Material). This transformation can be accomplished through appropriate selection of materials for the bilayer-support assembly so that the bottom layer expands with respect to the top layer and supporting structure when exposed to a suitable stimuli. This programmed response to external stimuli in the form of mismatch strain may eventually invert the curved bilayer and result in overall deformation of the assembly as shown in Figure 1(b). For our investigation, we considered a simple two dimensional model (shown in Figure 1(c) and discussed in section 2.1) that is an idealization of the structure shown in Figure 1(a). As the assembly responds to applied stimuli, different structural aspects affect its shape transformation behavior. Moreover, the expansion of the bottom layer imparts compressive strain on it due to the presence of the top layer and the supporting structures. This action might incite mechanical instabilities that can alter the assembly response by inducing additional deformation mechanisms. Our objectives were to determine how different structural parameters of the model affect the assembly response and define possible curved bilayer deformation mechanisms for a given mismatch strain. The design principles proposed by this work will contribute to 
the rapidly evolving field of stimuli responsive structures that have wide applications in fields as diverse as sensors, optics, microfluidics, soft robotics, microfabrication, smart surfaces, artificial muscles, energy harvesting, biomedical devices and origami inspired designs [19]-[21].

We start by describing the geometric details of the model and the essential aspects of our adopted computational scheme in section 2. The results of our analysis are presented in section 3. In that section, we identify the key design parameters and their effects on the deformation behavior of the mismatch strain- programmed curved bilayer-flexible support assembly. We present simple physical arguments to explain the trends in our results. In section 4, we discuss significant aspects of our modelling approach and observed deformation mechanisms, and possible implications of our results.

\section{Methods}

\subsection{Geometry}

In our analysis, we considered a simplified two dimensional version of the image shown in Figure 1 (a). Our model included a single curved beam attached to elastic supports at their ends. As shown in Figure 1(c), the curved beam consists of two layers, a top layer of thickness $t_{\text {top }}$ (colored in blue) that is insensitive to the applied stimuli and a bottom layer of thickness $t_{\text {bottom }}$ (colored in orange) that expands in response to the stimuli and eventually causes the bilayer to invert.

In Figure 1(a), the single layer curved beams were obtained from the buckling of an undeformed rectangular two dimensional structure. This single layer corresponds to the top layer of our model shown in Figure 1(c). The stimuli responsive bottom layer, which is required for beam inversion and an integral part of our model, could be deposited below the top layer either before or after the buckling procedure. For the curved shape, we considered a buckling scenario where both ends of the rectangular block were simply supported. The transverse displacement $w(\mathrm{X})$ along the length of the beam for the first buckling mode under simply supported boundary conditions has been given as [22]:

$$
w(X)=A \sin \left(\frac{\pi X}{L}\right) \quad ; \quad 0 \leq X \leq L
$$

Where, $X$ is measured along the length of the beam, $L$ is the length of the undeformed beam and $A$ is a constant whose value is not uniquely defined. Higher values of $A$ would represent more strongly curved buckled configuration and vice versa. We denote the length of the curved bilayer beam as $L_{\text {bilayer }}$.

Our model also includes two cantilever beams attached to the ends of the curved bilayer. These cantilevers are idealization of the movable support structures in Figure 1(a), that can bend during bilayer inversion. During the stimuli responsive swelling of the bottom layer, a moment $M_{\text {bilayer }}$ causes the bilayer to invert. Since the bilayer is attached to the cantilevers at its end, $M_{\text {bilayer }}$ is balanced by an equal and opposite moment $M_{\text {cantilever }}$ at the ends. $M_{\text {cantilever }}$ can be expressed as:

$$
M_{\text {cantilever }}=\frac{E_{\text {cantilever }} I_{\text {cantilever }}}{L_{\text {cantilever }}} \theta_{\text {cantilever }}
$$


Where, $E_{\text {cantilever }}$ is the Young's modulus, $I_{\text {cantilever }}$ is the area moment of inertia, $L_{\text {cantilever }}$ is the length and $\theta_{\text {cantilever }}$ is the angular deformation of the cantilever beam due to the action of $M_{\text {bilayer }}$. The term $\frac{E_{\text {cantilever }} I_{\text {cantilever }}}{L_{\text {cantilever }}}$ is often collectively referred as the cantilever beam bending stiffness, $K_{\text {cantilever }}$ and can be changed by modifying any of the three parameters involved. Similar to $K_{\text {cantilever }}$, inherent bending stiffness of the bilayer $K_{\text {bilayer }}$ can be defined as $\frac{(E I)_{\text {bilayer }}}{L_{\text {bilayer }}}$ for a weakly curved beam [23]. We calculated $(E I)_{\text {bilayer }}$ through the transformed section method [24] and used $K_{\text {bilayer }}$ to nondimensionalise the values of supporting cantilever bending stiffness (referred as normalized stiffness, $K_{\text {normalized }}=\frac{K_{\text {cantilever }}}{K_{\text {bilayer }}}$ ) and strain energy of the curved bilayer-flexible support assembly. Nondimensional parameter incorporating inherent bending stiffness values of the individual beam components has been reported in the literature [25] as it serves to determine the boundary of different deformation mechanisms. On the other hand, $K_{\text {bilayer }}$ is a natural candidate to non-dimensionalise the bending dominated strain energy (in context to our work) of any given beam as the energy can be expressed as $\frac{M^{2}}{\frac{2 E I}{L}}$ (where $\frac{E I}{L}$ is the beam bending stiffness and $M$ is the moment that acts on the beam) [26]. A detailed list of all the parameters and how they combine to form different dimensionless groups are shown in the Supplementary Material. An important aspect of the geometry shown in Figure 1(c) is the contact between the surfaces $S_{\text {bottom }}$ and $S_{\text {cantilever }}$ that occurs during the swelling induced inversion of the buckled beam.

\subsection{Finite Element Analysis}

We used the commercially available finite element package Abaqus/Standard [27] to numerically investigate the bilayer inversion scenario under different circumstances. We used 8-node biquadratic plane strain quadrilateral elements with reduced integration and did a mesh refinement study to determine the optimum mesh size. We investigated the stimuli responsive shape transformation of the bilayersupport assembly through swelling of the bottom layer by studying an equivalent thermal expansion problem. Specifically, we assigned a hypothetical value of thermal expansion coefficient to the bottom layer while keeping the remaining part of the model insensitive to temperature change. This procedure has been reported in the literature for simulating volumetric expansion of crystalline Silicon during lithiation [28]-[30] and $\mathrm{pH}$ dependent swelling-shrinking behavior of hydrogel [31]. We applied mismatch strains in small increments by increasing the temperature of the whole structure to investigate the shape transformation in a quasi-static scenario. Due to symmetry of our model shown in Figure 1(c), we simulated half of the model to reduce computational cost. For modeling the interaction between the constituent layers of the curved bilayer and that between the supporting cantilever beam and the bilayer, we used tie constraint for merging surfaces at the respective interfaces. Our finite element model also included contact between the surfaces $S_{\text {bottom }}$ and $S_{\text {cantilever }}$ to accurately capture their interaction during the swelling of the stimuli responsive bottom layer. We used the hard contact pressure-overclosure relationship with finite sliding and node to surface discretization method to minimize the penetration of $S_{\text {bottom }}$ into $S_{\text {cantilever }}$ To include the nonlinear effects of large displacements, we activated the nonlinear 
geometry option and used Newton's method for solving the equilibrium equations in our simulations. To account for the instabilities arising due to large displacements, we used an adaptive automatic stabilization scheme that employs spatially varying damping factors throughout the structure to eliminate rigid body modes without affecting the solution.

\subsection{Materials}

In our simulations, we used linear elastic constitutive relationship for the support cantilever beam and the curved bilayer materials. Single layer curved beams with necessary supporting structures have been fabricated from a wide range of linear elastic materials such as $\mathrm{SiO}_{2}$ [32], $\mathrm{Ni}, \mathrm{Au}$, Polyimide and SU8 [17] and Si [33]. We chose SU8, a photodefinable epoxy, to be the top layer and used an elastic modulus of $E_{S U 8}=4.02 \mathrm{GPa}$ and Poisson's ratio of $v_{S U 8}=0.22$ [17] for this material. As discussed earlier, the supporting cantilever beam possesses bending stiffness and hence resists the inversion of the curved bilayer beam. In our analysis, we systematically changed the elastic modulus of the cantilever beam $E_{\text {cantilever }}$ to investigate the effects of varying bending stiffness on bilayer inversion and used a constant value of the Poisson's ratio $v_{\text {cantilever }}=0.3$. Our envisioned design of the bilayer curved beam requires a stimuli responsive material demonstrating swelling as the bottom layer. We used PDMS (polydimethylsiloxane) as the stimuli responsive material to simulate shape transformation of the curved bilayer-support assembly. We used an elastic modulus of $E_{P D M S}=3 \mathrm{MPa}$ and Poisson's ratio of $v_{P D M S}=$ 0.49 for PDMS [34]. It is worth mentioning at this point that, specific values of the material properties are only used for carrying out the finite element simulations. The basic governing equations of elasticity and the principle of virtual work that underlie the formulation of the finite element method are independent of material properties and structural geometric configurations. Hence, the trends observed in our presented results could be reproduced through other material combinations as long as $\frac{E_{S U 8}}{E_{P D M S}}, v_{S U 8}$ and $v_{P D M S}$ remain the same.

\section{$\underline{3 \text { Results }}$}

\subsection{Bilayer Beam Curvature Inversion}

To understand how the thickness ratio of the bilayer for a given geometry affects the mismatch strain required for its curvature inversion, we considered several cases where the top layer thickness, $t_{\text {top }}$ was kept constant and the bottom layer thickness, $t_{\text {bottom }}$ was varied. We used the scheme shown in Figure S.2 in the Supplementary Materials to determine the mismatch strain required for the curved bilayer inversion.

As shown in Figure 2, the mismatch strain required to invert a curved bilayer depends strongly on the thickness ratio of the bilayer and the bending stiffness of the supporting cantilever beam. The results show that, for a given value of the cantilever bending stiffness $K_{\text {cantilever }}$, there exists a particular thickness ratio at which the mismatch strain required for inverting a curved bilayer is minimum. This is due to the competition between the bending moment exerted by the curved bilayer, $M_{\text {bilayer }}$ and the inherent bending resistance of the curved bilayer $K_{\text {bilayer }}$. For a given curved bilayer, 
$M_{\text {bilayer }} \propto\left(\frac{t_{\text {bottom }}}{t_{\text {top }}}, \Delta \varepsilon\right)$ and $K_{\text {bilayer }} \propto t_{\text {top }}^{3}\left(1+\frac{t_{\text {bottom }}}{t_{\text {top }}}\right)^{3}$ where $\Delta \varepsilon$ is the applied mismatch strain. When $t_{\text {bottom }} \sim 0, M_{\text {bilayer }}$ is very low at low mismatch strain values and $K_{\text {bilayer }}$ dominates the assembly behavior as the top layer possesses some bending stiffness. High values of $\Delta \varepsilon$ are needed to make $M_{\text {bilayer }}$ high enough to invert the curved bilayer. In the other limiting case, when $t_{\text {bottom }} \sim \infty$, both the top and bottom layer contributes to the bending resistance $K_{\text {bilayer }}$ and it becomes very high. To overcome this high $K_{\text {bilayer }}, M_{\text {bilayer }}$ needs to be high which can only be accomplished at higher values of $\Delta \varepsilon$ for a given $\frac{t_{\text {bottom }}}{t_{\text {top }}}$. In between the two extremes, there exists an intermediate thickness ratio where both the $K_{\text {bilayer }}$ and the $M_{\text {bilayer }}$ are balanced in a manner such that the bilayer inversion is possible at a relatively low value of mismatch strain, $\Delta \varepsilon$. As the supporting cantilever bending stiffness $K_{\text {cantilever }}$ becomes higher, the $M_{\text {bilayer }}$ needed to overcome the resistance to inversion is also higher, requiring a higher value of $\Delta \varepsilon$, as shown in Figure 2.

\subsection{Effects of Cantilever Bending Stiffness}

To investigate how the supporting cantilever beam bending stiffness affects the curved bilayer inversion scenario, we analyzed curved bilayers with a given thickness ratio attached to supporting cantilever beams of varying bending stiffness. For convenience, we varied the cantilever bending stiffness by changing its elastic modulus $E_{\text {cantilever }}$, although other parameters such as $I_{\text {cantilever }}$ and $L_{\text {cantilever }}$ could also be changed to achieve the same results. Moreover we use $K_{\text {normalized }}$ instead of $K_{\text {bilayer }}$ to remove any dependence of the results on our choice of numerical values for the structural parameters. We first investigate the change of deformation modes through studying the evolution of strain energy with increasing mismatch strain.

As shown in Figure 3, the strain energy stored in the assembly (consisting of the curved bilayer and the supporting structure) increases with increasing mismatch strain. The strain energy also depends on the normalized stiffness, $K_{\text {normalized }}$ and hence on the supporting cantilever beam bending stiffness, $K_{\text {cantilever }}$ .-For very low $K_{\text {normalized }}$ (i.e., very soft support cantilever), strain energy increases monotonically with mismatch strain and the bilayer smoothly changes to its inverted configuration. On the other hand, for very high $K_{\text {normalized }}$, the strain energy also increases monotonically but the bending moment in curved bilayer cannot overcome the $K_{\text {cantilever }}$ to assume an inverted configuration. Instead, creases form on the bottom surface- $\mathrm{S}_{\text {bottom }}$ of the bilayer at higher values of the mismatch strain $\Delta \varepsilon$. At intermediate values of $K_{\text {normalized }}$, the structures experience limit point snap-through as the strain energy drops sharply at a critical value of the mismatch strain $\Delta \varepsilon_{\text {critical }}$ and the assembly rapidly changes its configuration. The critical mismatch strain required for snap-through and the corresponding energy drop increase with increasing values of $K_{\text {normalized }}$. These behaviors are later discussed in more detail. Figures S.3 - S.5 show the gradual shape transformation of the bilayer for varying $K_{\text {normalized }}$ values. 
To understand how the critical mismatch strain required for snapping depends on $K_{\text {normalized }}$, we plot the $\Delta \varepsilon_{c r i t i c a l}$ as a function of the bilayer thickness ratio $\frac{t_{b o t t o m}}{t_{t o p}}$. As shown in Figure S.6, the critical strain for snapping, $\Delta \varepsilon_{\text {critical }}$, increases with increasing $K_{\text {normalized }}$ for a given $\frac{t_{\text {bottom }}}{t_{\text {top }}}$. The snapping window is bigger for bilayers with lower $\frac{t_{\text {bottom }}}{t_{\text {top }}}$ as they demonstrate snapping at lower values of $K_{\text {normalized }}$. For bilayers with thickness ratio $\frac{t_{\text {bottom }}}{t_{\text {top }}}$ higher than about 7.5 , the critical strain for snapping $\Delta \varepsilon_{\text {critical }}$ remains nearly constant for a wide range of thickness ratio. The result for thickness ratio $\frac{t_{b o t t o m}}{t_{\text {top }}}=5$, however, is an outlier.

We also develop deformation mechanism diagrams involving the curved bilayer thickness ratios, normalized bending stiffness and deformation modes. We gradually vary the cantilever bending stiffness $K_{\text {cantilever }}$ to change $K_{\text {normalized }}$ for different thickness ratios $\frac{t_{b o t t o m}}{t_{t o p}}$ and calculate the corresponding drop in strain energy to identify the conditions for gradual flipping, snapping and creasing. As shown in Figure $4(a-b)$, for a given thickness ratio $\frac{t_{b o t t o m}}{t_{t o p}}$, there exists two critical values of the normalized bending stiffness $K_{\text {normalized }}$, that bound the snapping behavior of the assembly. Below the lower bound or above the upper bound of the critical $K_{\text {normalized }}$ values, no energy drop occurs as gradual flipping or creasing takes place with increasing mismatch strain. The drop in strain energy (characterizing snapping) occurs at intermediate values of $K_{\text {normalized }}$. Within the snapping window of a given bilayer system, the amount of energy drop increases gradually with increasing $K_{\text {normalized }}$ (Figure 4(a)). This can be attributed to higher $\Delta \varepsilon_{\text {critical }}$ requirements for snapping with increasing values of $K_{\text {normalized }}$ as shown in Figure S.6. Higher $\Delta \varepsilon_{\text {critical }}$ means that the structure can store and hence release a higher amount of strain energy as it undergoes the snap-through instability. Our model reveals that, for bilayer systems with thickness ratio $\frac{t_{\text {bottom }}}{t_{\text {top }}}$ above 10 , both the peak energy drop and two critical values of $K_{\text {normalized }}$ are weakly dependent on the thickness ratios. But the behavior for lower values of thickness ratio $\frac{t_{\text {bottom }}}{t_{\text {top }}}$ is different, as shown in Fig. 4(a,b).

\subsection{Effects of Initial Curvature}


To investigate how the initial curvature of the curved bilayer affects the mismatch strain required for its inversion, we considered two curved geometries with $\frac{A}{L_{\text {bilayer }}}$ values of 0.05 and 0.1 . We construct a plot similar to Figure 2 by varying the thickness ratio $\frac{t_{\text {bottom }}}{t_{\text {top }}}$ for a $K_{\text {cantilever }}$ of $0.002 \mu \mathrm{N}$. $\mu \mathrm{m}$. This plot is given in the Supplementary Material as Figure S.7. Figure S.7 shows that strongly curved beams require higher mismatch strain for inversion at a given value of the thickness ratio $\frac{t_{b o t t o m}}{t_{t o p}}$. This can be explained by considering that the inherent bending resistance of the curved bilayer $K_{\text {bilayer }}$ is high for a bilayer with high initial curvature $\kappa$. Consequently, $M_{\text {bilayer }}$ needs to be high to overcome the bending resistance for a given value of the supporting cantilever bending stiffness $K_{\text {cantilever }}$. For bilayers with a given thickness ratio $\frac{t_{\text {bottom }}}{t_{\text {top }}}, M_{\text {bilayer }}$ can only be increased by increasing the mismatch strain $\Delta \varepsilon$, as shown in Figure S.7.

\section{Discussion and Conclusion}

In this work, we investigated a class of shape transformation induced by mismatch strain in a curved bilayer-flexible support assembly. We report the effects of parameters such as the bilayer thickness ratio, supporting structure bending stiffness and bilayer initial curvature on the bilayer curvature inversion and instabilities during shape transformation. We assigned linear elastic constitutive law to materials of the curved bilayer-support structure assembly. While this is an accurate representation of the behavior demonstrated by the passive top layer and supporting structures reported in the literature, stimuli responsive soft materials that constitute the bottom layer of our model, such as PDMS [35] and Hydrogel [36], often exhibit hyperelastic behavior. Treating the bottom layer as a hyperelastic solid in our finite element framework may be a better approximation. While we acknowledge this limitation of our study, we believe that a more realistic model involving nonlinear stress-strain relationship for the stimuli responsive bottom layer would have resulted in minor quantitative difference. Qualitatively, we should obtain similar mismatch strain-deformation mechanism behaviors reported in this work.

We observed snap through of the curved bilayer-supporting structure assembly under certain geometric conditions and supporting structure bending stiffness values. Snap through behavior has been observed both in nature [37] and in engineered structures such as shallow truss [38], MEMS beam [39], hemispherical dome [40] and jumping micro devices [41]. Snap through is a mechanical instability arising from nonlinear response characteristics of a structure. Often described in a parameterized control-state response setup [42], snap through occurs at a limit point along the static equilibrium path of a conservative system. At the limit point, the structural stiffness becomes zero and the structure undergoes large displacement as it jumps from one equilibrium configuration to another through passing an unstable phase [43]. The rapid change in structural configuration during snap through is often associated with significant release of stored strain energy. As stated in 2.2, our utilized stabilization scheme for investigating the bilayer-cantilever beam assembly employs artificial damping that absorbs the released strain energy and eventually stabilizes the system [27]. For confirming the accuracy of our solutions, we compared viscous forces with overall forces of the system and found that the former is several order of 
magnitudes smaller and hence has negligible effects. Our results demonstrated a limited snapping window, meaning that at a critical value of the normalized stiffness $K_{\text {normalized }}$, the assembly with a given thickness ratio $\frac{t_{\text {bottom }}}{t_{\text {top }}}$ undergoes a transition from gradual flipping to snapping. This can physically be understood by considering the energetics of the assembly. Below the lower bound of critical $K_{\text {normalized }}$, the stiffness of the support cantilever is so low that the assembly can undergo gradual change of curvature with increasing mismatch strain $\Delta \varepsilon$, storing the elastic energy only in the bilayer. Increasing the support stiffness $K_{\text {cantilever }}$ (and hence $K_{\text {normalized }}$ for a given $\frac{t_{\text {bottom }}}{t_{\text {top }}}$ ) restricts the assembly's deformation and hence increases its capability to store strain energy at a given $\Delta \varepsilon$ (Figure 3 and Figure S.3-S.5 of the Supplementary Material). At the lower bound critical value of $K_{\text {normalized }}$, the strain energy of the assembly reaches a local maximum at a critical mismatch strain $\Delta \varepsilon_{\text {critical }}$. At $\Delta \varepsilon_{\text {critical }}$, the assembly cannot sustain additional $\Delta \varepsilon$ and needs to release a portion of stored strain energy (snap-through) to recover its load carrying capabilities. Snap-through happens more vigorously owing to increased energy release as the $K_{\text {normalized }}$ approaches the upper bound critical value.

In our analysis, creases formed on the free surface of the stimuli responsive bottom layer for very high values of the normalized stiffness $K_{\text {normalized }}$. Creasing patterns are evident in a variety of natural systems including primate brain, human palm, infant arm and geological strata [44]. Creasing is an elastic instability that can occur on the free surfaces of a soft material under compressive loading [45]. Beyond a critical value of the applied compressive strain, creasing surfaces come into self-contact with each other while forming a sharp tip just below the surface [46]. Compared to limit point snap through instability that demonstrates the presence of an energy barrier, creasing instability is characterized by the presence of a bifurcation point where two equilibrium paths intersect. Consequently, there is no energy barrier as a creased surface and its corresponding flat counterpart have the same potential energy at a given applied strain [47]. Nonlinear finite element techniques have been used to simulate the initiation and subsequent growth of creasing instability on incompressible Neo-Hookean solids under plane strain compression [48]. Jin et. al. used carefully designed defects to pinpoint the location of crease initiation without affecting the critical strain requirements and enabled self-contact for subsequent folding of the creasing surfaces [49]. As the main objective of this work is to study the mismatch strain requirement for the shape transformation of a curved bilayer-supporting beam assembly and not to perform a detailed investigation of any particular deformation mode such as creasing, we did not carry out an elaborated analysis of crease formation. Instead, we maintained the same protocol mentioned in section 2.2 for the cases where creasing were observed. As we did not include any imperfection on the free surface of the bottom layer (as reported in the literature), generation of critical strain and subsequent crease initiation on this surface was arbitrary. But we were able to see no drop in strain energy and localization of high strain fields near the location of crease initiation as shown in Figure S.5, which are indicators of the creasing instability [46]. Our model predicts that, irrespective of the thickness ratio $\frac{t_{b o t t o m}}{t_{t o p}}$, creasing first occurs at the upper bound critical value of $K_{\text {normalized }}$. Figure 4(b) shows a mechanism map of the three deformation mechanisms, gradual flipping, snapping, and creasing, in the system property space, obtained from our simulation results. 
We considered a scenario where curved bilayers are attached to cantilever beams at their ends in a two dimensional geometric setup. The cantilevers function as elastic supports with tunable bending stiffness as they can deform during the stimuli driven inversion of the curved bilayer. Inversion of curved beams under mechanical loading has been studied extensively in the literature. Curved beam deformation behavior due to bending moment has been modelled using analytical [50], finite element [51] and experimental [52] techniques. Owing to their unique geometry, these structures often undergo bifurcation buckling and snap-through instability when the translational and rotational degrees of freedom are constrained at their ends [53]. As snap-through behavior is often characterized by the presence of a secondary equilibrium configuration and can potentially reduce actuation force requirements, curved beams have also been studied by the MEMS community. Using residual stress in a bilayer arising due to thermal expansion mismatch, planar micro-machined beams can be buckled to a curved configuration [32]. Researchers have investigated the snap-through and subsequent bi-stability of curved MEMS beams under mechanically applied point [33], [54] and distributed loading [55]-[57] conditions. Mechanically actuated bi-stable behavior of curved beams with end constraints have been utilized in applications as diverse as sensors, actuators, valves, optical/ electrical switches, digital micro-mirror devices, mechanical memory and vibration energy harvesting systems across multiple length scales [39], [55], [58]. Our work on curved beam-flexible support assembly proposes stimuli responsive programmable bilayer response as a potential replacement of mechanical actuation and thus complements the existing literature. Moreover, as our analyzed structure demonstrates snap-through (which indicates the presence of a secondary equilibrium configuration and hence bi-stability) under specific conditions and it is possible to fabricate these structures with a number of different material systems [17], existing applications reported in the literature can be realized and enhanced through the curved bilayer-flexible support assembly described in this work.

In conclusion, we numerically investigated mismatch strain induced inversion of a curved bilayer attached to flexible supports. We considered a simple two dimensional plane strain model that captures the essential features of a complex 3D architecture previously reported in the literature. Our model revealed that the curved bilayer inversion induced by mismatch strain depends strongly on the thickness ratio of the constituent layers, the bending stiffness of the supporting structure and the initial curvature. We proposed a non-dimensional stiffness incorporating the linear bending rigidity expressions of the flexible support and the curved bilayer. Our results showed that, the bilayer undergoes gradual flipping, snapping and creasing for very low, intermediate and very high values of the proposed non-dimensional stiffness. Snapping and creasing are two distinct types of mechanical instability phenomena where the former indicates rapid shape transformation and the later indicates formation of localized folds with self-contact. These findings imply that, the stimuli responsive behavior of the curved bilayer could be tuned and subsequently optimized to meet specific functional requirements through the modification of its structural

characteristics. The design principles reported in this work may be used to engineer sensors, actuators for traditional and soft robotics, electronic device components, metamaterials, energy storage and harvesting devices with on-demand functional capabilities.

\section{$\underline{\text { Acknowledgement }}$}

We acknowledge the support from the U.S. Department of Energy, Office of Science, Basic Energy Sciences, under Award \# DE-FG02-07ER46471. 


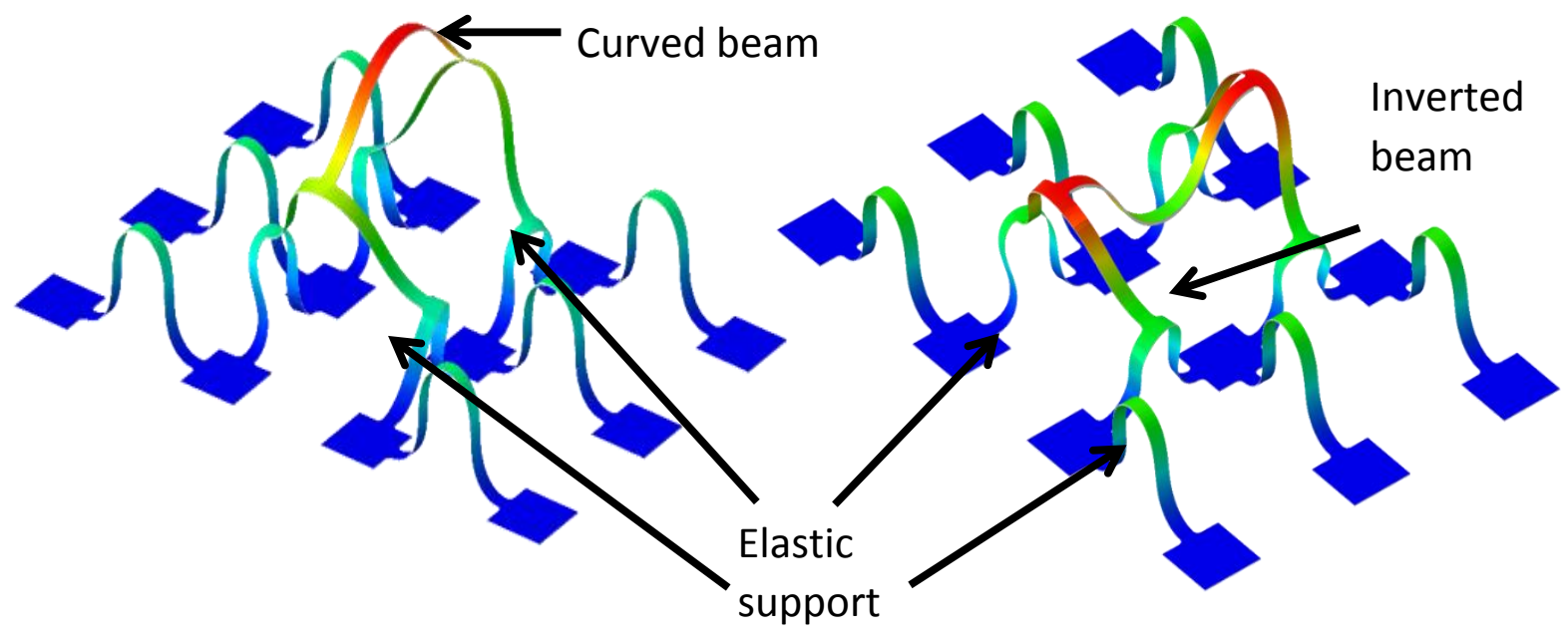

(a)

(b)

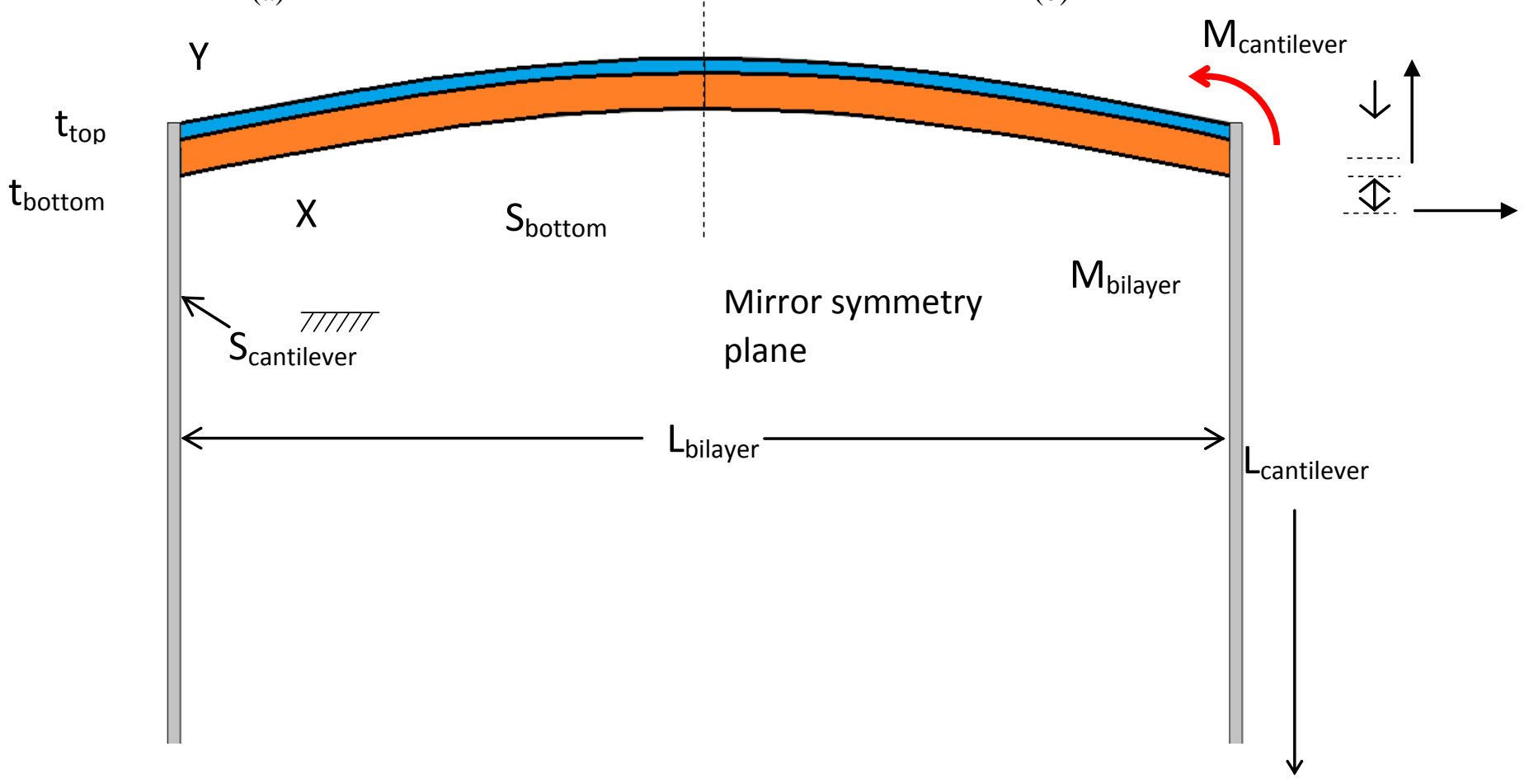


(c)

Figure 1: (a) 3D curved beam-flexible elastic support assembly as fabricated. (b) Possible inverted configuration of the curved bilayer and associated deformation of the assembly. The colors represent extent of the deformation (blue represents low and red represents high). (c) 2D simplified model considered in this work with movable end supports.

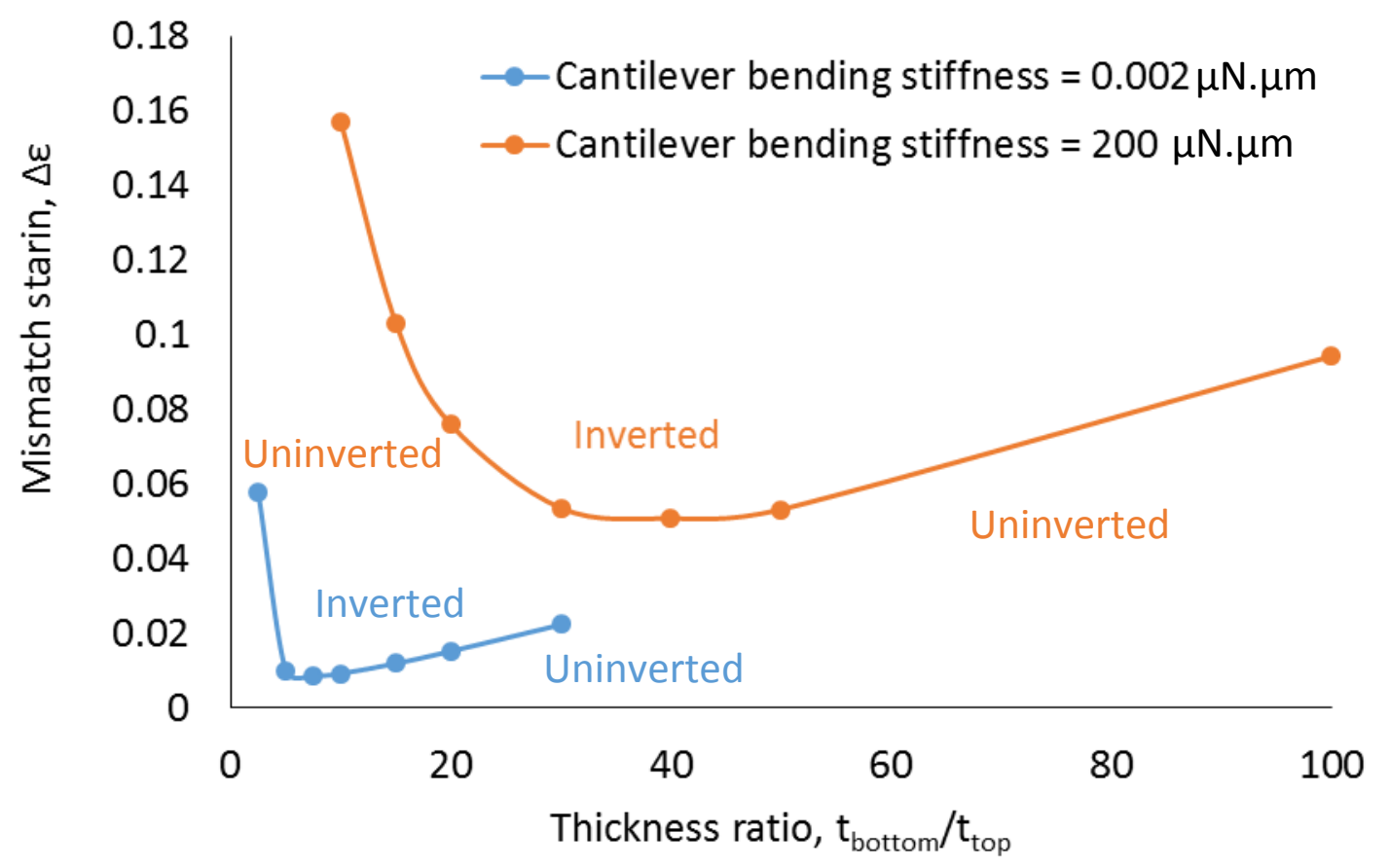

Figure 2: Mismatch Strain vs. Thickness ratio for varying bending stiffness of the supporting cantilever beam. This plot was made with a curved bilayer geometry having $\frac{A}{L_{\text {bilayer }}}=0.05$. 


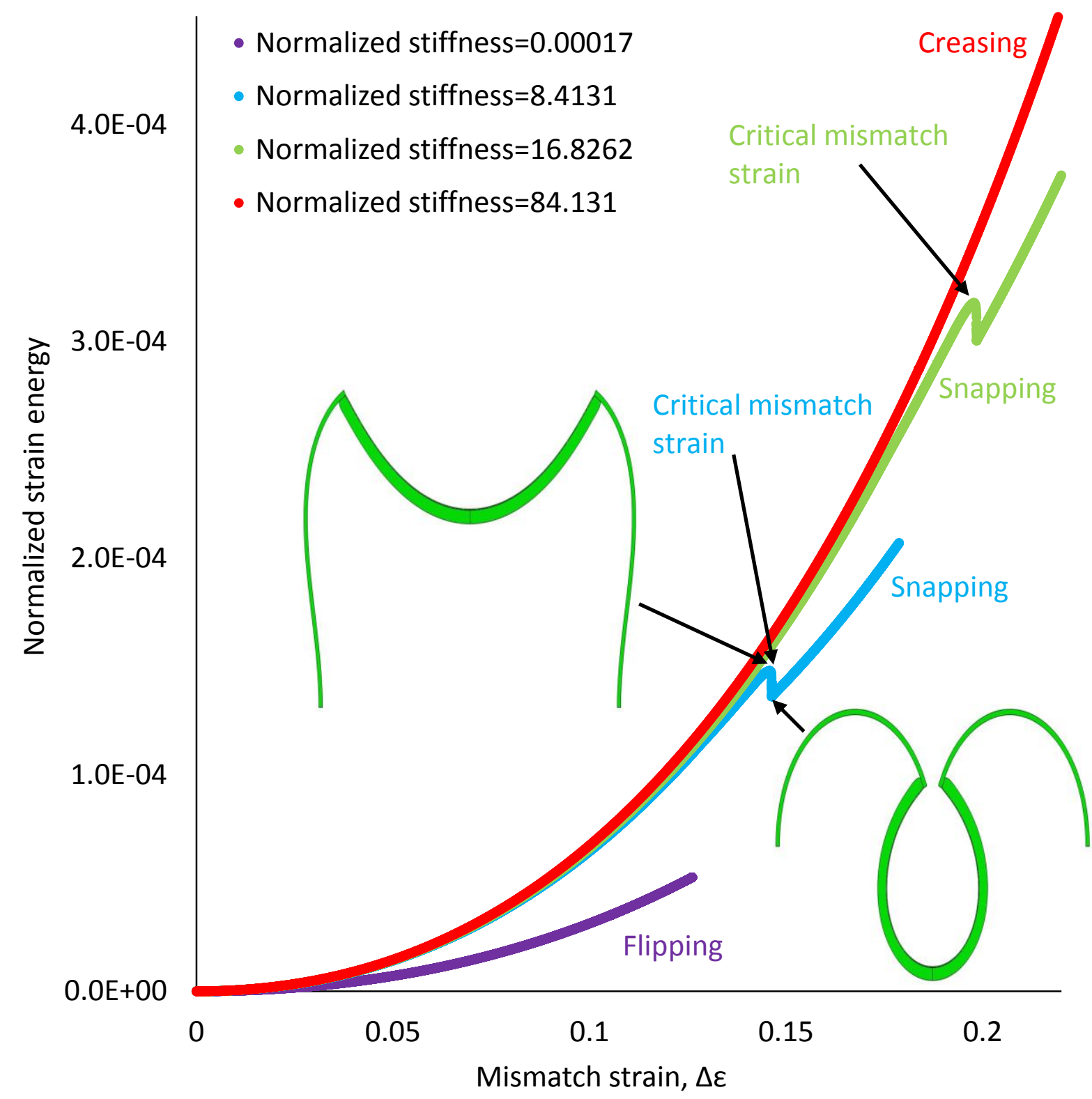

Figure 3: Normalized strain energy vs. Mismatch strain plot for varying normalized stiffness values of the supporting cantilever beam. This plot was made with a curved bilayer geometry having normalized curvature $\frac{A}{L_{\text {bilayer }}}=0.05$ and a thickness ratio $\frac{t_{\text {bottom }}}{t_{\text {top }}}=10$. 


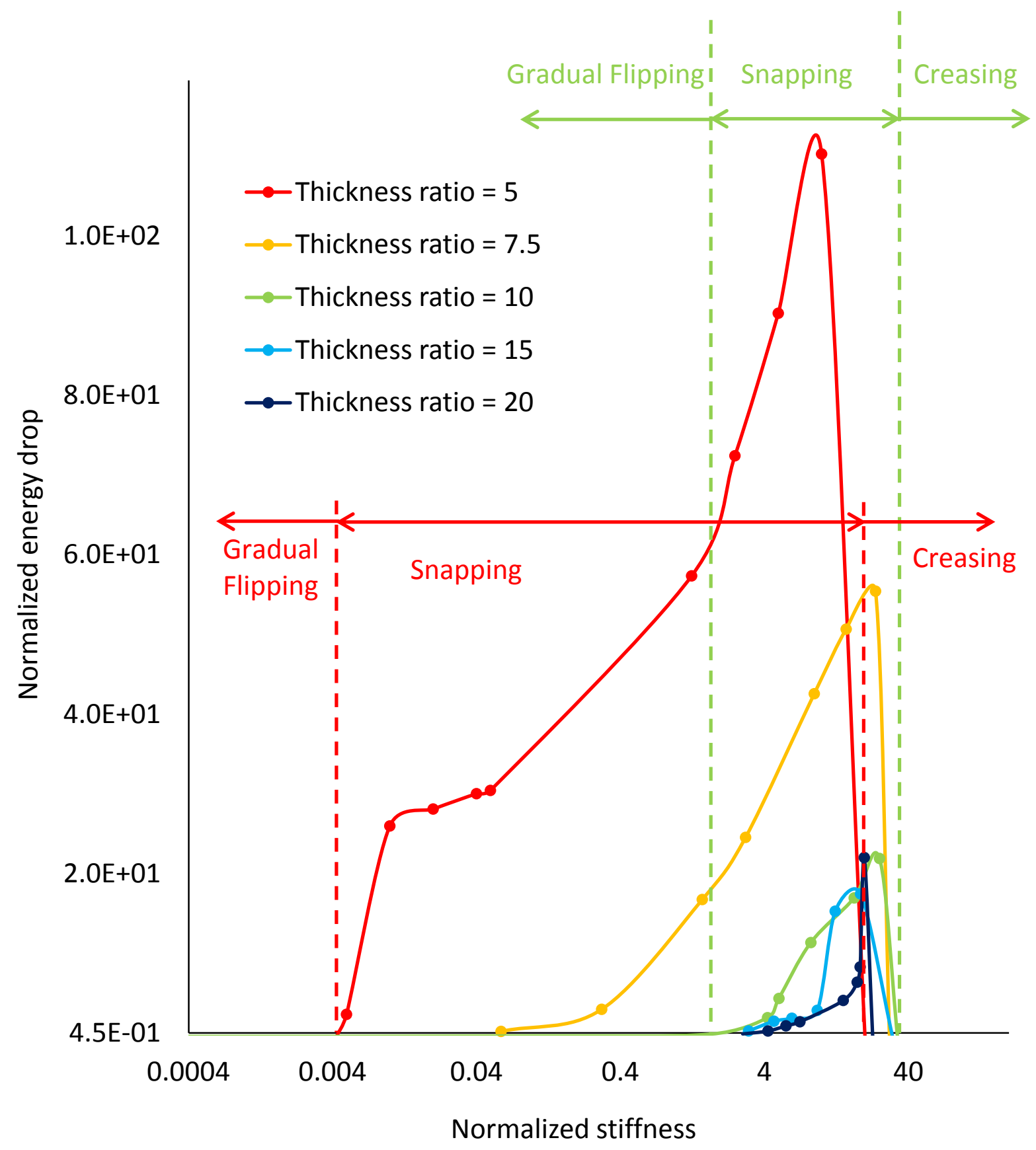

(a) 


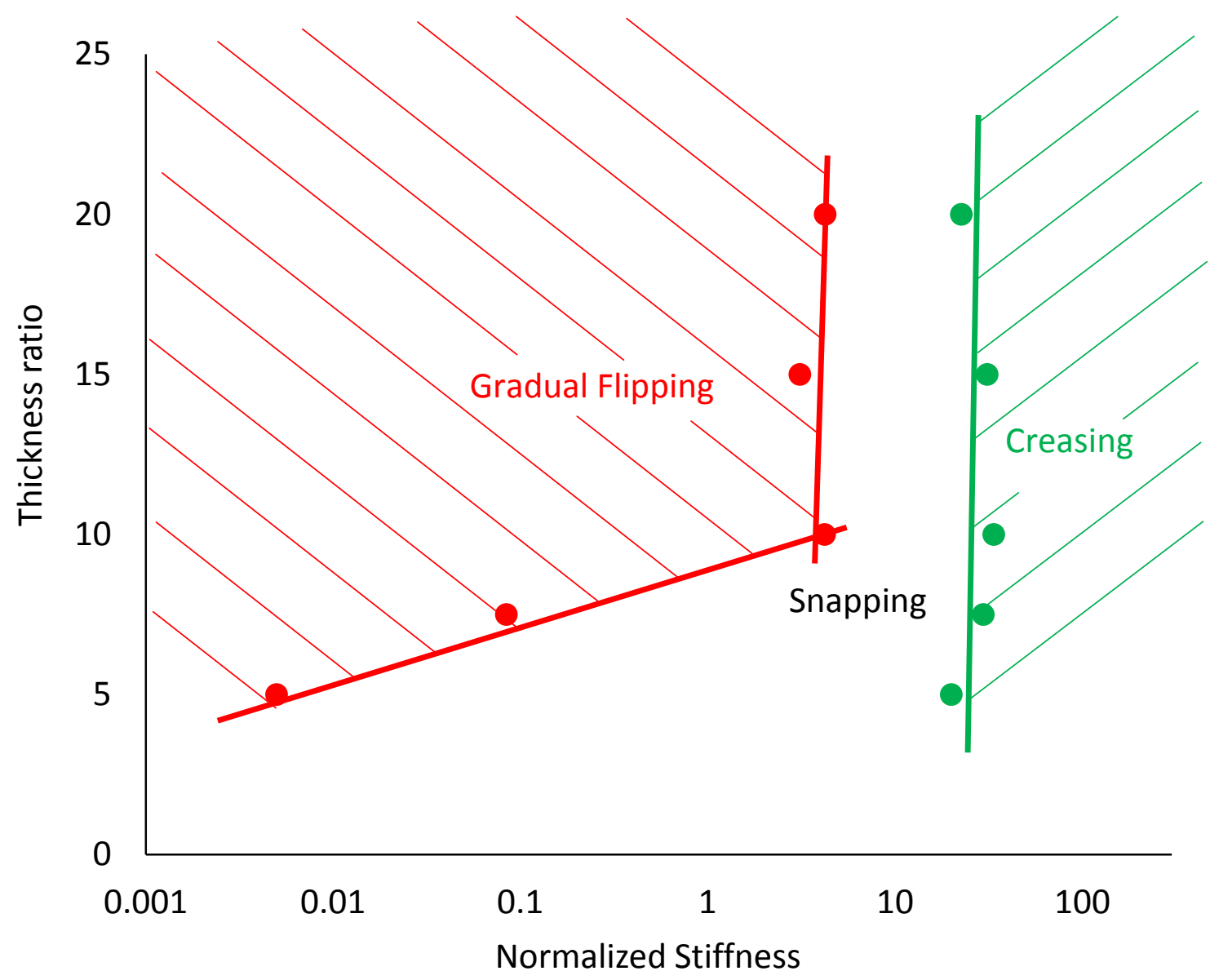

(b)

Figure 4: Phase diagram connecting curved bilayer thickness ratio, normalized stiffness and possible deformation modes. These plots were made with a curved bilayer geometry having a normalized curvature of $\frac{A}{L_{\text {bilayer }}}=0.05$. (a) shows the effects of thickness ratios and normalized stiffness values on the snap-through behavior. (b) shows the effects of thickness ratios on the critical stiffness values that bound the snapping behavior. 


\section{$\underline{\text { References }}$}

[1] S. J. Gerbode, J. R. Puzey, A. G. McCormick, and L. Mahadevan, "How the Cucumber Tendril Coils and Overwinds," Science, vol. 337, no. 6098, pp. 1087-1091, Aug. 2012.

[2] C. Llorens, M. Argentina, Y. Bouret, P. Marmottant, and O. Vincent, "A dynamical model for the Utricularia trap," J. R. Soc. Interface, vol. 9, no. 76, pp. 3129-3139, Nov. 2012.

[3] R. Elbaum, L. Zaltzman, I. Burgert, and P. Fratzl, "The Role of Wheat Awns in the Seed Dispersal Unit," Science, vol. 316, no. 5826, pp. 884-886, May 2007.

[4] E. Katifori, S. Alben, E. Cerda, D. R. Nelson, and J. Dumais, "Foldable structures and the natural design of pollen grains," Proc. Natl. Acad. Sci., vol. 107, no. 17, pp. 7635-7639, Apr. 2010.

[5] A. G. Volkov, J. C. Foster, T. A. Ashby, R. K. Walker, J. A. Johnson, and V. S. Markin, "Mimosa pudica: Electrical and mechanical stimulation of plant movements," Plant Cell Environ., vol. 33, no. 2, pp. 163-173, Feb. 2010.

[6] I. Burgert and P. Fratzl, "Plants control the properties and actuation of their organs through the orientation of cellulose fibrils in their cell walls," Integr. Comp. Biol., vol. 49, no. 1, pp. 69-79, Jul. 2009.

[7] M. Jamal, A. M. Zarafshar, and D. H. Gracias, "Differentially photo-crosslinked polymers enable self-assembling microfluidics," Nat. Commun., vol. 2, p. 527, Nov. 2011.

[8] C. Yoon, R. Xiao, J. Park, J. Cha, T. D. Nguyen, and D. H. Gracias, "Functional stimuli responsive hydrogel devices by self-folding," Smart Mater. Struct., vol. 23, no. 9, p. 094008, Sep. 2014.

[9] W. Huang, S. Koric, X. Yu, K. J. Hsia, and X. Li, "Precision Structural Engineering of Self-Rolledup 3D Nanomembranes Guided by Transient Quasi-Static FEM Modeling," Nano Lett., vol. 14, no. 11, pp. 6293-6297, Nov. 2014.

[10] J.-H. Na, A. A. Evans, J. Bae, M. C. Chiappelli, C. D. Santangelo, R. J. Lang, T. C. Hull, and R. C. Hayward, "Programming Reversibly Self-Folding Origami with Micropatterned PhotoCrosslinkable Polymer Trilayers," Adv. Mater., vol. 27, no. 1, pp. 79-85, Jan. 2015.

[11] T. Deng, C. Yoon, Q. Jin, M. Li, Z. Liu, and D. H. Gracias, "Self-folding graphene-polymer bilayers," Appl. Phys. Lett., vol. 106, no. 20, p. 203108, May 2015.

[12] V. B. Shenoy and D. H. Gracias, "Self-folding thin-film materials: From nanopolyhedra to graphene origami," MRS Bull., vol. 37, no. 09, pp. 847-854, Sep. 2012.

[13] K. Malachowski, J. Breger, H. R. Kwag, M. O. Wang, J. P. Fisher, F. M. Selaru, and D. H. Gracias, "Stimuli-Responsive Theragrippers for Chemomechanical Controlled Release," Angew. Chem. Int. Ed., vol. 53, no. 31, pp. 8045-8049, Jul. 2014.

[14] Z. L. Wu, M. Moshe, J. Greener, H. Therien-Aubin, Z. Nie, E. Sharon, and E. Kumacheva, "Threedimensional shape transformations of hydrogel sheets induced by small-scale modulation of internal stresses," Nat. Commun., vol. 4, p. 1586, Mar. 2013.

[15] M. J. Motala, D. Perlitz, C. M. Daly, P. Yuan, R. G. Nuzzo, and K. J. Hsia, "Programming matter through strain," Extreme Mech. Lett., vol. 3, pp. 8-16, Jun. 2015.

[16] M. E. McConney, A. Martinez, V. P. Tondiglia, K. M. Lee, D. Langley, I. I. Smalyukh, and T. J. White, "Topography from Topology: Photoinduced Surface Features Generated in Liquid Crystal Polymer Networks," Adv. Mater., vol. 25, no. 41, pp. 5880-5885, Nov. 2013.

[17] S. Xu, Z. Yan, K.-I. Jang, W. Huang, H. Fu, J. Kim, Z. Wei, M. Flavin, J. McCracken, R. Wang, A. Badea, Y. Liu, D. Xiao, G. Zhou, J. Lee, H. U. Chung, H. Cheng, W. Ren, A. Banks, X. Li, U. Paik, R. G. Nuzzo, Y. Huang, Y. Zhang, and J. A. Rogers, "Assembly of micro/nanomaterials into complex, three-dimensional architectures by compressive buckling," Science, vol. 347, no. 6218, pp. 154-159, Jan. 2015.

[18] Y. Zhang, Z. Yan, K. Nan, D. Xiao, Y. Liu, H. Luan, H. Fu, X. Wang, Q. Yang, J. Wang, W. Ren, H. Si, F. Liu, L. Yang, H. Li, J. Wang, X. Guo, H. Luo, L. Wang, Y. Huang, and J. A. Rogers, "A mechanically driven form of Kirigami as a route to 3D mesostructures in micro/nanomembranes," Proc. Natl. Acad. Sci., vol. 112, no. 38, pp. 11757-11764, Sep. 2015. 
[19] Q. Zhao, J. W. C. Dunlop, X. Qiu, F. Huang, Z. Zhang, J. Heyda, J. Dzubiella, M. Antonietti, and J. Yuan, "An instant multi-responsive porous polymer actuator driven by solvent molecule sorption," Nat. Commun., vol. 5, Jul. 2014.

[20] T. S. Kelby, M. Wang, and W. T. S. Huck, "Controlled Folding of 2D Au-Polymer Brush Composites into 3D Microstructures," Adv. Funct. Mater., vol. 21, no. 4, pp. 652-657, Feb. 2011.

[21] L. Ionov, "Hydrogel-based actuators: possibilities and limitations," Mater. Today, vol. 17, no. 10, pp. 494-503, Dec. 2014.

[22] F. P. Beer, Ed., Mechanics of materials, 6th ed. New York: McGraw-Hill, 2011.

[23] S. Timoshenko and J. M. Gere, Theory of elastic stability, 2nd ed., Dover ed. Mineola, N.Y: Dover Publications, 2009.

[24] A. Pytel and F. L. Singer, Strength of materials, 4th ed. New York: Harper \& Row, 1987.

[25] C. Xia, H. Lee, and N. Fang, "Solvent-driven polymeric micro beam device," J. Micromechanics Microengineering, vol. 20, no. 8, p. 085030, Aug. 2010.

[26] J. M. Gere and S. Timoshenko, Mechanics of materials, 4th ed. Boston: PWS Pub Co, 1997.

[27] ABAQUS (2014), “ABAQUS Documentation.” Dassault Systemes, Providence, RI, USA.

[28] H. Yang, F. Fan, W. Liang, X. Guo, T. Zhu, and S. Zhang, "A chemo-mechanical model of lithiation in silicon," J. Mech. Phys. Solids, vol. 70, pp. 349-361, Oct. 2014.

[29] M. Pharr, K. Zhao, X. Wang, Z. Suo, and J. J. Vlassak, "Kinetics of Initial Lithiation of Crystalline Silicon Electrodes of Lithium-Ion Batteries," Nano Lett., vol. 12, no. 9, pp. 5039-5047, Sep. 2012.

[30] J. Liu, N. Li, M. D. Goodman, H. G. Zhang, E. S. Epstein, B. Huang, Z. Pan, J. Kim, J. H. Choi, X. Huang, J. Liu, K. J. Hsia, S. J. Dillon, and P. V. Braun, "Mechanically and Chemically Robust Sandwich-Structured C@Si@C Nanotube Array Li-Ion Battery Anodes,” ACS Nano, vol. 9, no. 2, pp. 1985-1994, Feb. 2015.

[31] E. Epstein, J. Yoon, A. Madhukar, K. J. Hsia, and P. V. Braun, "Colloidal Particles that Rapidly Change Shape via Elastic Instabilities," Small, p. n/a-n/a, Oct. 2015.

[32] W. Fang and J. A. Wickert, "Post buckling of micromachined beams," J. Micromechanics Microengineering, vol. 4, no. 3, pp. 116-122, Sep. 1994.

[33] M. T. A. Saif, “On a tunable bistable MEMS-theory and experiment," J. Microelectromechanical Syst., vol. 9, no. 2, pp. 157-170, Jun. 2000.

[34] I. D. Johnston, D. K. McCluskey, C. K. L. Tan, and M. C. Tracey, "Mechanical characterization of bulk Sylgard 184 for microfluidics and microengineering," J. Micromechanics Microengineering, vol. 24, no. 3, p. 035017, Mar. 2014.

[35] T. Tallinen, J. Y. Chung, J. S. Biggins, and L. Mahadevan, "Gyrification from constrained cortical expansion," Proc. Natl. Acad. Sci., vol. 111, no. 35, pp. 12667-12672, Sep. 2014.

[36] Z. Wei, Z. Jia, J. Athas, C. Wang, S. R. Raghavan, T. Li, and Z. Nie, "Hybrid hydrogel sheets that undergo pre-programmed shape transformations," Soft Matter, vol. 10, no. 41, pp. 8157-8162, Sep. 2014.

[37] Y. Forterre, J. M. Skotheim, J. Dumais, and L. Mahadevan, "How the Venus flytrap snaps," Nature, vol. 433, no. 7024, pp. 421-425, Jan. 2005.

[38] G. Hrinda, "Snap-Through Instability Patterns in Truss Structures," presented at the 51st AIAA/ASME/ASCE/AHS/ASC Structures, Structural Dynamics, and Materials Conference, Orlando, FL; United States, 2010.

[39] M. D. Williams, F. van Keulen, and M. Sheplak, "Modeling of Initially Curved Beam Structures for Design of Multistable MEMS," J. Appl. Mech., vol. 79, no. 1, p. 011006, 2012.

[40] A. Madhukar, D. Perlitz, M. Grigola, D. Gai, and K. Jimmy Hsia, "Bistable characteristics of thickwalled axisymmetric domes," Int. J. Solids Struct., vol. 51, no. 14, pp. 2590-2597, Jul. 2014.

[41] H. Lee, C. Xia, and N. X. Fang, "First jump of microgel; actuation speed enhancement by elastic instability," Soft Matter, vol. 6, no. 18, p. 4342, 2010.

[42] C. Felippa, Nonlinear Finite Element Methods. University of Colorado Boulder. 
[43] R. Wiebe, L. Virgin, I. Stanciulescu, and S. Spottswood, "On Snap-Through Buckling," presented at the 52nd AIAA/ASME/ASCE/AHS/ASC Structures, Structural Dynamics and Materials Conference, Denver, Colorado, 2011.

[44] E. Hohlfeld and L. Mahadevan, "Unfolding the Sulcus," Phys. Rev. Lett., vol. 106, no. 10, Mar. 2011.

[45] L. Jin and Z. Suo, "Smoothening creases on surfaces of strain-stiffening materials," J. Mech. Phys. Solids, vol. 74, pp. 68-79, Jan. 2015.

[46] S. Cai, D. Chen, Z. Suo, and R. C. Hayward, "Creasing instability of elastomer films," Soft Matter, vol. 8, no. 5, pp. 1301-1304, 2012.

[47] Q. Wang and X. Zhao, "Phase Diagrams of Instabilities in Compressed Film-Substrate Systems," $J$. Appl. Mech., vol. 81, no. 5, p. 051004, Dec. 2013.

[48] R. Zhao, T. Zhang, M. Diab, H. Gao, and K.-S. Kim, "The primary bilayer ruga-phase diagram I: Localizations in ruga evolution," Extreme Mech. Lett., vol. 4, pp. 76-82, Sep. 2015.

[49] L. Jin, A. Auguste, R. C. Hayward, and Z. Suo, "Bifurcation Diagrams for the Formation of Wrinkles or Creases in Soft Bilayers," J. Appl. Mech., vol. 82, no. 6, p. 061008, Jun. 2015.

[50] L. Mentrasti, "Bending of large curvature beams.," Int. J. Solids Struct., vol. 38, no. 32-33, pp. 5703-5726, Aug. 2001.

[51] W. Yu, D. H. Hodges, V. Volovoi, and C. E. S. Cesnik, "On Timoshenko-like modeling of initially curved and twisted composite beams," Int. J. Solids Struct., vol. 39, no. 19, pp. 5101-5121, Sep. 2002.

[52] R. M. Guedes and A. Sá, "Numerical analysis of singly curved shallow composite panels under three-point bend load," Compos. Struct., vol. 83, no. 2, pp. 212-220, Apr. 2008.

[53] Y.-L. Pi, M. A. Bradford, and B. Uy, "In-plane stability of arches," Int. J. Solids Struct., vol. 39, no. 1, pp. 105-125, Jan. 2002.

[54] J. Qiu, J. H. Lang, and A. H. Slocum, "A Curved-Beam Bistable Mechanism," $J$. Microelectromechanical Syst., vol. 13, no. 2, pp. 137-146, Apr. 2004.

[55] S. Park and D. Hah, "Pre-shaped buckled-beam actuators: Theory and experiments," Sens. Actuators Phys., vol. 148, no. 1, pp. 186-192, Nov. 2008.

[56] J. S. Han, J. S. Ko, and J. G. Korvink, "Structural optimization of a large-displacement electromagnetic Lorentz force microactuator for optical switching applications," J. Micromechanics Microengineering, vol. 14, no. 11, pp. 1585-1596, Nov. 2004.

[57] J. S. Han, J. S. Ko, Y. T. Kim, and B. M. Kwak, "Parametric study and optimization of a microoptical switch with a laterally driven electromagnetic microactuator," J. Micromechanics Microengineering, vol. 12, no. 6, pp. 939-947, Nov. 2002.

[58] B. Andò, S. Baglio, A. R. Bulsara, and V. Marletta, "A bistable buckled beam based approach for vibrational energy harvesting,” Sens. Actuators Phys., vol. 211, pp. 153-161, May 2014. 